\title{
Resultados de la implementación del proyecto La formación práctico-experimental en las Ciencias Naturales
}

Implementation results of the project "The practical-experimental training in the natural sciences"

\section{Volumen 18, Número 3}

Setiembre-Diciembre

pp. 1-30

\section{Este número se publica el 1 de setiembre de 2018}

DOI: https://doi.org/10.15517/aie.v18i3.33446

\author{
Andrés Israel Yera Quintana \\ Micaela Castillo Estenoz \\ Iraida Pérez Hernández \\ Erismelkys Espinosa Castillo
}

Revista indizada en REDALYC, $\underline{\text { SCIELO }}$

Revista distribuida en las bases de datos:

LATINDEX, DOAJ, REDIB, IRESIE, CLASE, DIALNET, SHERPA/ROMEO, QUALIS-CAPES, MIAR

Revista registrada en los directorios:

ULRICH'S, REDIE, RINACE, OEI, MAESTROTECA, PREAL, CLACSO 


\title{
Resultados de la implementación del proyecto La formación práctico-experimental en las Ciencias Naturales
}

Implementation results of the project "The practical-experimental training in the natural sciences"

\author{
Andrés Israel Yera Quintana ${ }^{1}$ \\ Micaela Castillo Estenoz \\ Iraida Pérez Hernández \\ Erismelkys Espinosa Castillo
}

\begin{abstract}
Resumen: El artículo presenta los resultados de la implementación del proyecto La formación prácticoexperimental en las Ciencias Naturales, en el proceso de enseñanza-aprendizaje de los preuniversitarios del municipio Ciego de Ávila, y en la licenciatura en Educación en las referidas especialidades. Concretamente, se introdujeron, en la práctica, la concepción teórica asumida sobre formación práctico-experimental (FPE) y la metodología para contribuir con ella. Al ser una investigación de corte mixto, se evaluaron ocho indicadores en cuatro categorías. Las evaluaciones fueron realizadas al inicio, en dos momentos intermedios, y al final de la implementación del proyecto, a partir del análisis integrado de las informaciones obtenidas por la aplicación de los instrumentos diseñados y de acuerdo con los criterios establecidos. Se realizó un muestreo intencional que abarcó a 294 participantes, pertenecientes a las cinco unidades de estudio determinadas. Se constataron avances en todos los indicadores evaluados, entre los que resaltan los referidos a niveles de socialización y aplicación de los resultados del proyecto. Las mediciones intermedias permitieron el perfeccionamiento y ajuste de las acciones de implementación y favorecieron una evaluación final más efectiva y pertinente. En general, se corroboraron avances en la calidad de los trabajos teórico-prácticos y seminarios, en los resultados de la evaluación del aprendizaje y en las preparaciones de las asignaturas. Asimismo, se advierte la satisfacción de estudiantes, docentes, técnicos y directivos educacionales respecto al mejoramiento del desempeño de sus funciones.
\end{abstract}

Palabras clave: formación, desarrollo de las habilidades, experimento (en clase), aprendizaje, enseñanza de las ciencias naturales.

\begin{abstract}
The article presents the results on the implementation of the project "The practical-experimental training in the natural sciences", in the teaching-learning process of the pre-university students of Ciego de Ávila municipality and in the degree in education in these specialties. Specifically, the theoretical conception assumed on the practical-experimental training (FPE) and the methodology to contribute to the said training were introduced in practice. As a mixed-case study, eight indicators were evaluated in four categories. The evaluations were carried out at the initial, two at the intermediate and final moments on the implementation of the project, based on the integrated analysis of the information obtained by the application of the designed instruments and according to the established criteria. An intentional sampling was carried out which included 294 participants, belonging to the five determined units of study. Progress was observed in all the indicators evaluated and notable among them those referring to the levels of socialization and application of the project results. The intermediate measurements allowed the improvement and adjustment of the implementation actions and favored a more effective and pertinent final finding. In general, progress was made in the quality of theoretical-practical work and seminars; the results of the evaluation of the learning process and the preparation of the subjects. Likewise, the satisfaction of students, teachers, technicians and educational managers regarding the improvement on the performance of their functions is noticed.
\end{abstract}

Key words: training, skills development, experiments (lessons), learning, teaching of naturals sciences.

\footnotetext{
1 Las personas autoras son docentes del Departamento de

Ciencias Naturales de la Universidad de Ciego de Ávila, Cuba.
}

Dirección electrónica de contacto: andreyq@sma.unica.cu

Artículo recibido: $1^{\circ}$ de noviembre, 2017

Enviado a corrección: 15 de febrero, 2018

Aprobado: 21 de mayo, 2018 


\section{Introducción}

El desarrollo de las ciencias naturales en los últimos años se encuentra en correspondencia con el auge de la ciencia en sentido general. El referido desarrollo influye notablemente en las asignaturas que se explican como parte del currículo escolar de los diferentes niveles educacionales, siendo el bachillerato y la educación superior, en los que se sistematizan y profundizan los contenidos y se prepara al futuro profesional para la obtención, en su momento, de nuevos resultados científicos.

A tono con lo anterior el Ministerio de Educación cubano (MINED) ha llevado a cabo el perfeccionamiento de los contenidos de los programas de las asignaturas y del proceso de enseñanza-aprendizaje (PEA), sustentado en la investigación científica. En el bachillerato, en particular, se ha intencionado dicho perfeccionamiento hacia la mejora sostenida de la calidad de la formación de su egresado. En ello han desempeñado un rol fundamental las asignaturas de ciencias naturales, en cuyos programas se declaran como exigencias didácticas esenciales la realización de demostraciones, experimentos, prácticas de laboratorios, excursiones, visitas a museos y centros científicos de la producción y de los servicios de la comunidad, lo que revela la visión integral de estas ciencias y favorece el fortalecimiento de una cultura científica en los estudiantes.

Para la consecución de tal fin el PEA de estas asignaturas debe procurar de manera constante, vías cada vez más efectivas, de estimulación de su estudio. Esencial resulta en ello la realización de actividades práctico- experimentales (APE) en la casa, en el laboratorio de la escuela, en las áreas experimentales y/o de práctica, a fin de favorecer el aprendizaje y la formación del estudiante y su desempeño eficiente en la solución de problemas docentes y de la vida.

En la búsqueda inicial de los referentes teóricos de la APE en la didáctica de las ciencias naturales se constató que son numerosos los investigadores del tema y variados los criterios que se han asumido. Dentro de los referentes considerados se encuentran (Baraqué, 1992; Cuétara, 2004; Rionda, 1999; Rojas y Achiong, 1990; Salcedo, et al., 2002; Zilberstein y Silvestre, 2004), los que abordaron la experimentación y/o las actividades prácticas en sus obras; aportaron definiciones y enfatizaron en su importancia, así como en el desarrollo de hábitos y habilidades.

Otros investigadores del tema (Alexandre, 2011; Banasco, et al., 2013; Colado, 2003; Hedesa, 2015; Iglesias, 2012; Martínez, 2009; Pérez y Hedesa, 2010; Rodríguez, 2009; Vidal, 2012), han aportado procedimientos para integrar formas de experimentos, 
fundamentado operaciones de habilidades específicas a desarrollar desde las actividades prácticas, entre otras contribuciones no menos importantes, no obstante, en la sistematización teórica de las aportaciones apuntadas llevada a cabo durante el desarrollo del proyecto, aún se evidencia un vacío teórico en sus referentes científico- pedagógicos, en particular, en explicitar la influencia de la APE en el modo de pensar y actuar de los estudiantes en correspondencia con el carácter de su participación en ella.

Con independencia de las aportaciones mencionadas y de los esfuerzos del MINED por perfeccionar el PEA se identificaron, mediante la observación y el intercambio realizado en los preuniversitarios, por un equipo de investigadores (como parte del proyecto de investigación "La formación práctico- experimental (FPE) en las ciencias naturales" asociado a un programa nacional) dificultades en la práctica, tanto en la enseñanza como en el aprendizaje de los estudiantes; estos últimos mostraron carencias en el dominio de los contenidos vinculados con las APE, escasa motivación por estas ciencias, poco desarrollo de habilidades y hábitos, limitadas posibilidades de aplicación del contenido y una participación esencialmente dirigida a la ejecución de las operaciones contenidas en las guías de las APE que les facilitan sus profesores, en las que sus opiniones y criterios han estado, en general, ausentes.

De la misma manera se constató que los docentes no muestran un accionar didáctico coherente durante la ejecución de la APE ni aprovechan todas las potencialidades del contenido y del desarrollo de los estudiantes para demostrar el vínculo teoría-práctica, lo que incide de manera notable en la FPE de los estudiantes.

Desde su inicio el proyecto de investigación trazó como objetivos:

1. Determinación de los fundamentos teóricos de la FPE en el PEA de las ciencias naturales.

2. Diseño de una metodología para contribuir a la FPE de los estudiantes en el PEA de las ciencias naturales.

3. Evaluación de los resultados de la implementación de la metodología para contribuir a la FPE de los estudiantes en las ciencias naturales del preuniversitario y la licenciatura en educación.

El presente artículo tiene como propósito divulgar los resultados obtenidos en la concreción del tercer objetivo declarado. Para ello se determinaron indicadores con sustento en los fundamentos teóricos que de la FPE se asumieron en el proyecto. 


\section{Referente teórico}

Para la consecución del primer objetivo del proyecto se sistematizó la teoría precedente de la didáctica de las ciencias naturales, en particular, lo referido a las actividades prácticas y/o experimentales. Una de las concepciones de base asumida en la sistematización realizada apunta que "La actividad práctica experimental no es la simple manipulación sino el equilibrio de la actividad motora e intelectual del alumno en la que se logra la familiarización, la observación e interpretación de los fenómenos químicos que son objeto de estudio" (Caballero y Vidal, 2014, p. 5).

Tomando como sustento las referidas concepciones se asumió inicialmente, que la FPE se entendía como:

(...) el proceso en el que se produce la apropiación de los contenidos de las ciencias naturales y se alcanza un desarrollo gradual en los estudiantes al implicarse e interactuar con los objetos, fenómenos y procesos, con el uso de los métodos de observación y la experimentación. (Castillo, et al., 2014, p. 10)

En la apuntada concepción inicial se resaltó el carácter de proceso de dicha formación, lo que presupone sistematicidad en la realización de las APE, gradación de sus niveles de dificultad y de independencia de los estudiantes, y la aproximación cada vez más intencionada de la observación y la experimentación a su concepción de métodos científicos y por tanto de desarrollo del pensamiento.

Resultado de los últimos análisis teóricos presentados y debatidos en las jornadas de trabajo científico del proyecto de investigación se consideró que la FPE en las ciencias naturales:

(...) es un estado dinámico del desarrollo de la personalidad del sujeto, que supone una forma de pensar y de actuar respecto al análisis de situaciones y la solución de problemas prácticos y/o experimentales en función de comprender y explicar los hechos, procesos y fenómenos de la naturaleza y la vida en permanente contrastación con la teoría existente. (Castillo, et al., 2014, p. 12).

Con total apego a las concepciones presentadas respecto a la FPE en las ciencias naturales y en correspondencia con la contribución que deben hacer sus contenidos, se precisaron algunas de las características que se entendieron como esenciales y que se manifiestan en un sujeto, las que apuntan a una adecuada FPE, ellas son: 
1. El reconocimiento de la necesidad y utilidad del contenido de las ciencias naturales para su adecuada interacción con el medio.

2. El conocimiento que demuestra de los aparatos conceptual, categorial y legal de las ciencias naturales.

3. Las valoraciones personales que realiza respecto a la necesidad y posibilidad de comprobación práctica y/o experimental de los fundamentos teóricos de las ciencias naturales.

4. Las habilidades y hábitos que demuestra para la gestión de la información científica actualizada respecto a los procesos, fenómenos y objetos que estudian las ciencias naturales.

5. Las habilidades que demuestra para el diseño de alternativas de comprobación práctico-experimental de los fundamentos teóricos de las ciencias naturales.

6. La organización y el desempeño que demuestra durante la puesta en práctica de las alternativas que diseña y su actuación mesurada ante inconvenientes que puedan ocurrir.

7. Las posibilidades que demuestra para concentrarse en lo esencial y formular conclusiones respecto a los resultados obtenidos, en correspondencia con la comprensión que alcanza de los fundamentos teóricos de las ciencias naturales y el diseño de alternativas concebido.

8. La valoración personal que realiza de sus logros y limitaciones individuales y de los colectivos alcanzados durante la puesta en práctica de las alternativas de comprobación práctico-experimental.

9. Las actitudes que asume y los valores que demuestra en su interacción con el medio.

Si bien las características declaradas no agotan todas las posibles, de acuerdo con lo complejo que es el PEA, y por demás la formación de la personalidad del sujeto, desde el punto de vista didáctico, apuntan a la necesidad de que durante el PEA de las ciencias naturales se estimule de manera permanente el desarrollo del pensamiento de los estudiantes y su amor por la ciencia, que se enfrenten a situaciones de aprendizaje en que sientan la necesidad de corroborar práctica y/o experimentalmente, en los casos posibles y de acuerdo con las condiciones existentes, los fenómenos, procesos y hechos que estudian, así como contrastar los resultados que obtienen con los fundamentos teóricos y aportes científicos de la ciencia en cuestión. 
Las consideraciones apuntadas cobran doble importancia en el estudiante de licenciatura en educación del área de las ciencias naturales, pues en la medida en que incorporen y manifiesten en su actuación las características arriba declaradas, podrán contribuir de manera más eficiente a la FPE de sus futuros estudiantes.

\section{Metodología}

Los métodos y las técnicas para el desarrollo de la investigación y la evaluación de los resultados de su introducción en la práctica, se seleccionaron en correspondencia con el tipo de investigación mixto, objetivo general y las tareas científicas formuladas (Hernández, Fernández y Baptista, 2010). Se emplearon básicamente del nivel teórico: el analíticosintético, la inducción-deducción, el histórico-lógico, los que fueron de utilidad para la precisión del marco teórico referencial. Se utilizaron del nivel empírico el análisis documental, la entrevista, la encuesta, la prueba pedagógica y la observación, mediante los cuales se pudo obtener la información necesaria de las instituciones del nivel preuniversitario del municipio Ciego de Ávila y de las carreras de licenciatura en educación en las especialidades de ciencias naturales de la Facultad de Ciencias Pedagógicas.

Los resultados científicos del proyecto que se introdujeron en la práctica fueron concretamente la concepción teórica asumida sobre FPE (definición operacional y características) y la metodología para contribuir a la referida formación en los estudiantes (contentiva de pasos lógicos y procederes didácticos).

La metodología concibió como un primer paso lógico la realización de un taller de socialización sobre las concepciones de FPE asumidas en el proyecto; dicho taller se desarrolló en dos contextos diferentes, uno de ellos con docentes, técnicos de laboratorio y directivos y el otro con estudiantes. Aunque las intenciones de los talleres fueron diferentes, el propósito fue el mismo: contribuir a la FPE de los estudiantes, en ello constituyó condición de base la actuación protagónica consciente de ellos.

Constituyeron además, pasos lógicos de dicha metodología: el diagnóstico de potencialidades, limitaciones y oportunidades de las condiciones, los contextos y el desarrollo de los estudiantes; el diseño de APE de acuerdo con las orientaciones de los programas de las asignaturas, los intereses de los estudiantes y las oportunidades del contexto; la ejecución de las APE diseñadas; la valoración de la calidad de la ejecución determinando logros y deficiencias, individuales y colectivas, sobre la base de los indicadores de la FPE; la elaboración de conclusiones a partir de la contrastación de los 
resultados obtenidos con los referentes teóricos de las ciencias; y la proyección de acciones individuales y colectivas para perfeccionar de manera sostenida la FPE.

La evaluación de los resultados de dicha introducción en la práctica fue organizada y dirigida por la responsable del proyecto de investigación del departamento de Ciencias Naturales en la facultad de Ciencias Pedagógicas y apoyada por los dos jefes del departamento, quienes facilitaron el desarrollo de las acciones y asignaron responsabilidades concretas a los docentes.

Las acciones desarrolladas para la introducción de los resultados del proyecto se concretaron en:

1. El intercambio con los involucrados en el estudio para obtener su consentimiento y disposición de participar en la experiencia, previa explicación de la necesidad de perfeccionar la APE de las ciencias naturales para favorecer una formación de mayor calidad del bachiller y del licenciado en educación en las referidas ciencias.

2. La capacitación de metodólogos y técnicos de laboratorio de ciencias naturales.

3. EI PEA de los preuniversitarios del municipio de Ciego de Ávila y de las carreras de licenciatura en educación en las especialidades Biología-Geografía y Biología-Química.

4. La superación profesional de docentes de ciencias naturales en diferentes modalidades.

5. El trabajo metodológico en los diversos niveles colectivos.

6. La formación académica a través de la maestría en Didáctica de las Ciencias Naturales, el Doctorado en Ciencias Pedagógicas, y en el trabajo científico estudiantil.

Con la intención de dar seguimiento a la introducción de los resultados del proyecto se consideró necesaria la realización del monitoreo (García, 2010), el que permite la observación frecuente, la recolección y el análisis de la información acerca del funcionamiento de dicha introducción con respecto a lo planificado, lo que posibilita tomar decisiones para ajustar acciones, retroalimentar al proyecto, efectuar redistribución de recursos humanos o materiales y proponer acciones correctivas en dependencia de los objetivos planteados.

Según lo expresado, y en correspondencia con las particularidades de los beneficiarios de los resultados y su disposición para participar, se decidió realizar cuatro mediciones: una inicial comprendida en los meses septiembre y octubre de 2014 , dos intermedias en junio de 2015 y en febrero de 2016 y una final en noviembre de 2016. 
La medición inicial estuvo dirigida a obtener información sobre el inicio de la implementación en lo relativo a los aspectos organizativos, el compromiso mostrado por los implicados, el cumplimiento de las actividades del cronograma, la identificación de las barreras que pudieran limitar el cumplimiento del cronograma y la toma de decisiones al respecto, así como evaluar inicialmente los indicadores determinados.

Las mediciones intermedias se orientaron a la obtención de información sobre la concreción del resultado en las APE y su influencia en los docentes y en la FPE de los estudiantes, además de la identificación de las potencialidades, carencias, amenazas y a la compilación de evidencias durante el proceso de introducción.

La medición final tomó como base los criterios que desde el punto de vista teórico han ofrecido diversos investigadores en el contexto educacional. Así, la evaluación de los indicadores sirvió de guía para valorar en qué medida la introducción de los resultados del proyecto de investigación contribuyeron a la mejora de la calidad de los procesos formativos que se desarrollan en las instituciones educativas (Arnaiz, 2011; Rojas, 2014).

A partir de la asunción de los presupuestos expresados se procedió a la determinación de indicadores para evaluar los resultados de la introducción del proyecto, ellos fueron:

1. Calidad de los trabajos teórico- prácticos y seminarios.

2. Resultados de la evaluación del aprendizaje.

3. Satisfacción de los implicados con la realización de las APE.

4. Utilización de materiales docentes elaborados por el proyecto.

5. Calidad de las preparaciones de las asignaturas.

6. Nivel de aplicación del resultado.

7. Nivel de socialización de los resultados.

8. Apertura de nuevas líneas y/o tareas de investigación.

La posición teórica asumida exige la profundización en las metodologías existentes en el contexto educativo y para la evaluación de los resultados de la implementación del proyecto se siguió una metodología estructurada en cuatro etapas, la que permitió a los investigadores apropiarse de las acciones metodológicas acerca de cómo implementar la evaluación de los resultados de manera lógica y coherente (Rojas, 2014). Seguidamente se explicita la manera en que se organizó dicha metodología. 


\subsection{Primera etapa: diseño de la evaluación de los resultados}

a) Determinación del objetivo de la evaluación:

Evaluar las transformaciones ocurridas en el PEA de las ciencias naturales a partir de la introducción de los resultados científicos obtenidos en el proyecto "La FPE en las ciencias naturales".

b) Elección de las fuentes de obtención de información:

Los cuadernos de notas de los estudiantes seleccionados en la muestra, los informes de trabajos teórico-prácticos y seminarios, los instrumentos de evaluaciones aplicadas con carácter práctico, los planes de clases, el criterio de directivos, metodólogos y la familia. Asimismo, fotos y grabaciones de las APE.

c) Precisión de las técnicas e instrumentos indispensables a utilizar, bajo la premisa de no excederse para lograr la cooperación de los beneficiarios y no causar molestias e interferencias en los procesos que conducen:

Guía de revisión de planes de clases, guía de observación a clases, guía de revisión de cuadernos de notas y guía de entrevistas.

d) Selección de la muestra:

La población la constituyeron los docentes de ciencias naturales de la provincia de Ciego de Ávila, (los que se encuentran en el ejercicio de la profesión como docentes graduados y los que se encuentran en su formación de pregrado); los metodólogos municipales y provincial del área del saber; los directores de los institutos preuniversitarios urbanos (IPU); los técnicos de los laboratorios y los estudiantes del décimo grado de la provincia. La muestra fue seleccionada de manera intencional en el municipio cabecera homónimo y se estructuró en las unidades de estudio que se precisan en la Tabla 1. 
Tabla 1

Composición de la muestra por unidades de estudio

\begin{tabular}{|c|c|}
\hline Unidades de estudio & Muestra \\
\hline Docentes graduados & $\begin{array}{l}19 \text { (16 del departamento de Ciencias Naturales de la Facultad de } \\
\text { Ciencias Pedagógicas y } 3 \text { de los IPU) }\end{array}$ \\
\hline Técnicos de laboratorios & $\begin{array}{l}5 \text { ( } 2 \text { del departamento de Ciencias Naturales de la Facultad de } \\
\text { Ciencias Pedagógicas y } 3 \text { de los IPU) }\end{array}$ \\
\hline $\begin{array}{l}\text { Estudiantes del tercer año } \\
\text { de licenciatura en } \\
\text { educación }\end{array}$ & $\begin{array}{l}20 \text { (10 de la carrera de Biología-Geografía y } 10 \text { de la carrera de } \\
\text { Biología-Química correspondientes al departamento de Ciencias } \\
\text { Naturales de la Facultad de Ciencias Pedagógicas) }\end{array}$ \\
\hline Estudiantes de $10^{\circ}$ grado & 240 (de los tres IPU del municipio Ciego de Ávila) \\
\hline $\begin{array}{l}\text { Directivos: } \\
\text {-Jefes de departamentos }\end{array}$ & $\begin{array}{l}5 \text { ( } 2 \text { del departamento de Ciencias Naturales de la Facultad de } \\
\text { Ciencias Pedagógicas y } 3 \text { de los departamentos homónimos de los } \\
\text { IPU) }\end{array}$ \\
\hline $\begin{array}{l}\text {-Directores de IPU } \\
\text {-Metodólogos }\end{array}$ & $\begin{array}{l}3 \text { (del municipio Ciego de Ávila) } \\
2 \text { ( } 1 \text { del municipio Ciego de Ávila y la metodóloga provincial) }\end{array}$ \\
\hline $\begin{array}{c}\text { Total de unidades de } \\
\text { estudio: } 5\end{array}$ & Total de participantes: 294 \\
\hline
\end{tabular}

Fuente: Elaboración propia, 2017

e) Elaboración del cronograma de trabajo.

f) Determinación de los principales resultados a obtener, los que coinciden con los declarados como indicadores.

\subsection{Segunda etapa: organización del proceso evaluativo}

g) Responsabilización de un docente por cada asignatura del área para coordinar la comisión evaluadora.

h) Preparación de los evaluadores en el procedimiento de introducción de la metodología, en sus posibles alcances y en los contenidos de las diferentes etapas.

i) Responsabilización de un docente por asignatura del departamento de Ciencias Naturales de la Facultad de Ciencias Pedagógicas para la recogida de evidencias.

\subsection{Tercera etapa: ejecución del proceso de evaluación}

j) Análisis de las propuestas del proyecto en función de los espacios de aplicación de cada instrumento y su presentación a la institución educativa en que se aplican para convenirlas y/o realizar modificaciones en caso necesario y adoptar las medidas de aseguramiento.

k) Análisis de las formas de aplicación de los instrumentos diseñados.

I) Precisión de los instrumentos a aplicar. 
m) Realización de balances mensuales del comportamiento de la evaluación de los resultados y proposición de acciones para la mejora en caso necesario.

\subsection{Cuarta etapa: recopilación y procesamiento de la información; valoración y socialización de los resultados.}

n) Recolección de la información obtenida a partir de las diversas fuentes.

ñ) Procesamiento de la información recopilada y de las evidencias.

o) Análisis de la información obtenida.

p) Determinación de las fortalezas y debilidades encontradas y elaboración de plan de mejora para dar continuidad al perfeccionamiento del PEA de las asignaturas.

q) Elaboración del informe final.

r) Debate del informe final con todos los implicados en el proceso de implementación (en los IPU y en el departamento de Ciencias Naturales de la Facultad de Ciencias Naturales).

s) Presentación de los resultados finales de la evaluación en las direcciones municipal y provincial de Educación y en la Facultad de Ciencias Pedagógicas de la Universidad.

La implementación de los resultados del proyecto se efectuó durante los cursos 20142015 y 2015-2016 en la formación del bachiller en el municipio cabecera de la provincia Ciego de Ávila y en la formación de los licenciados en educación del área de las ciencias naturales.

Se entendió conveniente efectuar la valoración de las transformaciones ocurridas de manera separada por cada una de las asignaturas que componen el área en el sistema de educación cubano: Geografía, Biología y Química, de acuerdo con sus especificidades, la experiencia de los docentes que participaron en la introducción y evaluación de los resultados del proyecto, la organización escolar, las particularidades de los estudiantes de los IPU y de la licenciatura en educación.

\section{Resultados y su análisis}

\subsection{Resultados cuantitativos}

La medición de los indicadores determinados para la evaluación de los resultados de la implementación del proyecto se realizó a partir del análisis integrado de las informaciones y evidencias recogidas por la aplicación de las técnicas e instrumentos referidos en la metodología diseñada. Los indicadores fueron evaluados en las categorías de bastante 
adecuado (4), adecuado (3), poco adecuado (2) y no adecuado (1). Los criterios para otorgar las categorías se declaran en el Anexo 1.

En la tabla 2 se muestra la evaluación realizada.

Tabla 2

Evaluación categorizada de los indicadores según categorías de constatación

\begin{tabular}{|l|c|c|c|}
\hline Indicadores & $\begin{array}{c}\text { Constatación } \\
\text { Inicial }\end{array}$ & $\begin{array}{c}\text { Media de las } \\
\text { Constataciones } \\
\text { Intermedias }\end{array}$ & $\begin{array}{c}\text { Constatación } \\
\text { Final }\end{array}$ \\
\hline $\begin{array}{l}\text { I. Calidad de los trabajos teórico prácticos y } \\
\text { seminarios }\end{array}$ & 2 & 3 & 4 \\
\hline II. Resultados de la evaluación del aprendizaje & 2 & 3 & 4 \\
\hline $\begin{array}{l}\text { III. Satisfacción de los implicados con la } \\
\text { realización de las APE }\end{array}$ & 1 & 3 & 4 \\
\hline $\begin{array}{l}\text { IV. Utilización de materiales docentes elaborados } \\
\text { por el proyecto }\end{array}$ & 1 & 2 & 3 \\
\hline $\begin{array}{l}\text { V. Calidad de las preparaciones de las } \\
\text { asignaturas }\end{array}$ & 1 & $\mathbf{2}$ & 3 \\
\hline VI. Nivel de aplicación del resultado & 1 & $\mathbf{3}$ & 4 \\
\hline VII. Nivel de socialización de los resultados & 1 & 2 & 4 \\
\hline $\begin{array}{l}\text { VIII. Apertura de nuevas líneas y/o tareas de } \\
\text { investigación }\end{array}$ & 1 & 3 \\
\hline
\end{tabular}

Fuente: Elaboración propia, 2017

Los resultados mostrados en la tabla anterior se ilustran con mayor visibilidad en la figura 1.

Figura 1

Distribución comparativa de la evaluación de los indicadores según categorías de constatación

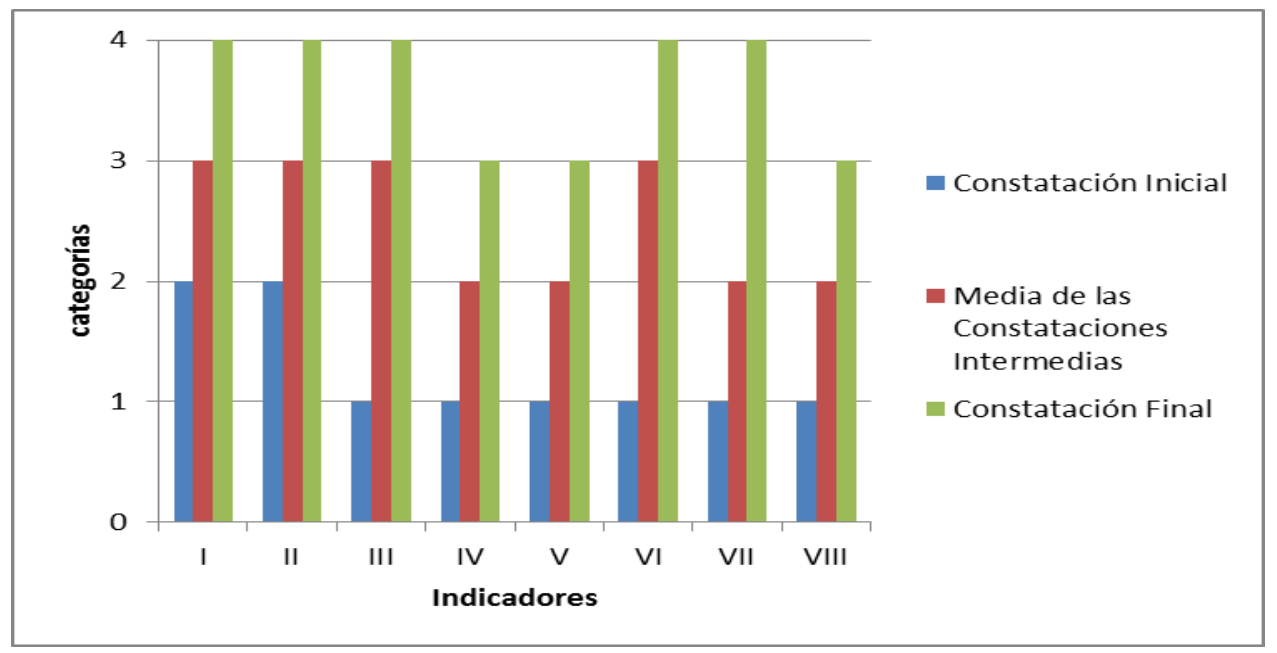

Fuente: Elaboración propia, 2017 
Se observa en la figura 1 que todos los indicadores muestran un incremento sostenido en las categorías establecidas desde la constatación inicial, en los momentos intermedios y hasta la constatación final. Los avances más notables se observan en los indicadores III, VI y VII, al ser los que comenzaron con las categorías más bajas y culminaron con la más alta de las establecidas.

\subsection{Resultados cualitativos}

4.2.1 Transformaciones logradas con la introducción de los resultados del proyecto en Biología

\section{Grupo I: en los preuniversitarios avileños}

Los resultados del proyecto fueron introducidos mediante el trabajo cooperado de investigadores del proyecto, los docentes de la asignatura en el décimo grado, los técnicos de laboratorio, estudiantes de la licenciatura en educación de las carreras Biología-Geografía y Biología-Química y sus tutores, durante el cumplimiento de su práctica laboral.

La implementación de los resultados se realizó a través del perfeccionamiento del PEA de la asignatura, en particular, en el rediseño de los sistemas de APE y de evaluación del aprendizaje y su puesta en práctica; el establecimiento de relaciones más sistemáticas entre la teoría y la práctica, al explicar los objetos de estudio de esta asignatura e incentivar la motivación por su estudio desde las clases y la realización de los trabajos teórico-prácticos y seminarios; el desarrollo de sociedades científicas estudiantiles y de un proyecto sociocomunitario.

Los seminarios se organizaron de forma tal que los estudiantes, a partir de la consulta de diferentes fuentes de información, indagaron sobre determinadas temáticas con especialistas en la enseñanza de la Biología y del sector de la salud. La búsqueda realizada los situó en condiciones de dar respuestas a diversas preguntas de aplicación y creación referidas a la relación de los virus con los organismos.

En el diseño de las guías de las APE y en la concepción de la preparación previa a su ejecución por los estudiantes, constituyeron condiciones de base el estudio de la información científica actualizada, la orientación sobre las posibilidades de comprobación práctica y la necesidad de sistematizar las operaciones que conforman las habilidades para lograr su dominio.

El diseño de los proyectos de evaluaciones parciales, tuvo como base el vínculo del contenido biológico con el medio natural y las actividades desarrolladas en el laboratorio, así 
se incluyeron como contenidos a evaluar las operaciones a seguir durante la observación del material biológico.

Para la realización de trabajos teórico-prácticos se organizaron equipos en correspondencia con los contenidos del programa de estudio. Cada uno de los equipos investigó los fundamentos teóricos del tema seleccionado y desarrolló una APE como vía de constatación empírica de su estudio teórico; en estas actividades se destacan experimentos de cómo hacer el vino y el yogurt, el proceso de fotosíntesis, la presencia de carbohidratos en las plantas y el transporte de sustancias. Durante la ejecución de dichas actividades se advirtieron como dificultades: pobre desarrollo de habilidades manipulativas y prevalencia de las acciones físicas sobre las intelectuales, por lo que fue pertinente insistir en la necesidad de entrenarse en el cuestionamiento de los resultados que se obtienen y contrastarlos con la teoría que los fundamenta, evitando de esta manera ser ejecutores inconscientes de operaciones del laboratorio.

Se crearon sociedades científicas estudiantiles con el tema "Experimentos de Ciencias Naturales que puedes hacer en casa" con la intención de fomentar el amor por la investigación y contribuir a la asimilación de los contenidos objeto de estudio. La conformación de dichas sociedades se realizó de acuerdo con la voluntariedad y disposición de participar de sus integrantes. Se elaboró un manual didáctico contentivo de doce actividades experimentales que pueden ser realizadas en la casa. El manual se puso a disposición de los estudiantes y fue consultado y aplicado por un grupo significativo de ellos, no obstante su utilización no fue sistemática. El uso del manual fue socializado en diferentes espacios educativos y publicado en las redes sociales.

\section{Grupo II: en la formación de los licenciados en educación}

La introducción de los resultados del proyecto en las disciplinas Botánica, Didáctica de la Biología y Microtecnia biológica, así como en la práctica laboral investigativa en el tercer año de la licenciatura en educación en las especialidades de Biología-Geografía y BiologíaQuímica, posibilitó:

1. El mejoramiento de la calidad de la preparación de las clases a partir de explicitar las operaciones de las habilidades en las clases teóricas y su sistematización en las clases prácticas, prácticas de laboratorio, seminarios y en las prácticas de campo. Ello demandó de la realización de varios talleres metodológicos y clases demostrativas para lograr una influencia más coherente y sistemática de todos los profesores de acuerdo con las concepciones asumidas por el proyecto. 
2. La elaboración de materiales de apoyo a la docencia que potenciaban el vínculo de la teoría con la práctica, tales como: guías de clases prácticas y prácticas de laboratorio las que contienen los fundamentos teóricos de las actividades prácticas, las normas de seguridad para el trabajo en el laboratorio, las acciones para el desarrollo de habilidades prácticas y la sistematización de las mismas sobre la base de la metodología establecida en el proyecto.

3. El desarrollo de habilidades como la obtención de muestras tanto microscópicas como macroscópicas, la observación de diferentes estructuras vegetales y la manipulación correcta del microscopio óptico. Para lograr este desarrollo fue esencial la comprensión por los estudiantes de la necesidad de sistematizar la ejecución de las operaciones en situaciones diversas, el estudio y preparación previa y la confrontación de los resultados que obtenían con el tratamiento de la teoría en la bibliografía científica actualizada. Este proceso demandó sistematicidad en la orientación didáctica intencionada dada la dualidad de la formación de los estudiantes como aprendices de las ciencias naturales y futuros profesores de ellas.

4. La publicación de cuatro artículos científicos relacionados con las actividades prácticas y el desarrollo de habilidades prácticas y experimentales desde las asignaturas en coautoría con sus tutores y la socialización de los resultados obtenidos en diversos eventos.

5. El tránsito gradual de los estudiantes de la dependencia total del profesor a la independencia relativa de él, durante la ejecución de APE, logrando establecer con mayor sistematicidad el vínculo de la teoría con la práctica y reconociendo la utilidad de lo aprendido.

6. La participación de cinco estudiantes de tercer año de la carrera Biología-Química, como parte de su trabajo científico estudiantil, en la organización pedagógica de Sociedades Científicas que sobre actividades prácticas se desarrollaron con estudiantes de Preuniversitario.

4.2.2 Transformaciones logradas con la introducción de los resultados del proyecto en Geografía

\section{Grupo I: en los preuniversitarios avileños}

Los resultados del proyecto, al igual que en Biología, fueron introducidos mediante el trabajo cooperado de investigadores del proyecto, los docentes de la asignatura en el décimo 
grado, los estudiantes de la licenciatura en educación de la carrera Biología-Geografía y sus tutores, durante el cumplimiento de su práctica laboral.

La implementación de los resultados se realizó a través del perfeccionamiento del PEA de la asignatura, en particular, en la propuesta de un conjunto de actividades prácticas y experimentales de acuerdo con las potencialidades del programa. Ello precisó de análisis rigurosos de la naturaleza de los contenidos de la asignatura y de las oportunidades de los contextos en que están enclavas la instituciones educativas, pues como tendencia general existía en los profesores la creencia infundada de que en escasos momentos se podían emplear actividades prácticas, y en mucha menor medida experimentales.

Los docentes de los tres institutos diseñaron y ejecutaron APE no contenidas en el programa a partir de las orientaciones recibidas por el proyecto, lo que favoreció el vínculo de la teoría con la práctica y la apropiación de procederes didácticos novedosos no abordados desde esta perspectiva en sus estudios de pregrado y postgrado.

La apropiación de esos procederes por los docentes contribuyó a que pudieran ofrecer una explicación más precisa y motivante a los fenómenos naturales y sociales en los que fue posible demostrar el vínculo de la teoría con la práctica y con situaciones de la vida cotidiana. Se evidenció un enriquecimiento en la preparación de la asignatura y una utilización adecuada de los materiales didácticos puestos a su disposición por el proyecto.

Los estudiantes alcanzaron resultados superiores en el aprendizaje de los contenidos evidenciados en el ejercicio de evaluación final de la asignatura cuya forma fue un seminario integrador de la unidad "Interacción naturaleza- sociedad". En dicho seminario la generalidad de los estudiantes demostraron interés por la investigación, situación que marca la diferencia con respecto a etapas anteriores. Su actuar estuvo caracterizado por una búsqueda, recopilación y procesamiento de la información de manera activa, consciente y con adecuada valoración, de acuerdo con su nivel, del rigor científico de las diversas fuentes utilizadas. A ello contribuyó la orientación sistemática de los profesores.

Durante el seminario pudieron ilustrar los procesos dañinos que afectan el medio ambiente a escala global, nacional y local; logrando identificar sus causas y consecuencias. Demostraron, mediante la realización de experimentos, los procesos de contaminación del agua, la contaminación atmosférica, la erosión del suelo y la deforestación, y a través de la elaboración de maquetas, pudieron explicar las consecuencias de ellos para la vida del hombre. 
Asimismo, mostraron satisfacción con la búsqueda efectuada y avanzaron de manera discreta en la identificación de los logros y las dificultades presentadas durante su preparación previa y la exposición de sus trabajos, aspecto este que se considera esencial desde la propia concepción teórica del proyecto pues resulta imprescindible que el estudiante, como manifestación de la FPE que alcanza, pueda autovalorar su desempeño y orientar su actuación posterior.

\section{Grupo II: en la formación de los licenciados en educación}

La introducción de los resultados del proyecto en las disciplinas Geografía Regional, Geografía Económica y Didáctica de la Geografía en el tercer año de la licenciatura en educación en la especialidad de Biología-Geografía posibilitó el desarrollo de habilidades de colectas de muestras de materiales geográficos tales como rocas y suelos, de la lectura de mapas, esencialmente las de ubicar, localizar y caracterizar geográficamente, las que demostraron durante la realización de las prácticas de estudio realizadas en Loma de Cunagua, Boquerón, área de reforestación de la Playita de Ciego de Ávila y Cayo Coco.

Desde la Didáctica de la Geografía y la práctica laboral investigativa fue posible el diseño de experimentos y la apropiación de contenidos topográficos y habilidades necesarias para la determinación de parcelas y poligonales, así como para la realización de las mediciones topográficas y su empleo para la toma de muestras en la naturaleza. El colectivo de profesores del tercer año dedicó numerosas sesiones de trabajo metodológico para incorporar y mantener de manera sostenida las concepciones teóricas asumidas sobre la FPE.

El diseño y la ejecución de APE por los estudiantes les permitió la profundización en el estudio de la localidad y demostrar el vínculo de la teoría con la práctica, así como explicar situaciones problemáticas de la vida cotidiana. Asimismo, demostraron domino de los contenidos para generalizar lo investigado en la localidad a otras áreas del territorio nacional y a escala global.

Se apropiaron del proceder para el análisis de los fenómenos, procesos físicos y económico- geográficos a partir de la identificación de los aspectos que intervienen de una manera u otra en ellos. 
4.2.3 Transformaciones logradas con la introducción de los resultados del proyecto en Química

\section{Grupo I: en los preuniversitarios avileños}

Los resultados del proyecto, al igual que en Biología y en Geografía, fueron introducidos, mediante el trabajo cooperado de investigadores del proyecto, los docentes de la asignatura en el décimo grado, los técnicos de laboratorio, estudiantes de la licenciatura en educación de la carrera Biología-Química y sus tutores, durante el cumplimiento de su práctica laboral.

La implementación de los resultados se realizó a través del perfeccionamiento del PEA de la asignatura, en particular, en el rediseño y puesta en práctica de los sistemas de APE y de evaluación del aprendizaje.

Concretamente se trabajó en la concepción y el desarrollo de las prácticas de laboratorio, demostraciones experimentales y experimentos de clase. Fue necesario demostrar a los estudiantes la importancia de una adecuada preparación previa a la realización de las APE y a las prácticas de laboratorio, en particular, e incentivar en ellos la motivación por el estudio del contenido teórico que sustenta dichas actividades, dadas las dificultades que han manifestado para comprender los resultados que obtienen en las experiencias que realizan y la ejecución de operaciones y procedimientos sin responder a una estructuración organizada de su actuación.

Se organizó un horario para el uso del laboratorio docente en los IPU en sesiones contrarias a las clases, de manera que los estudiantes pudieran asistir a ellos para que se familiarizaran con los útiles del laboratorio y desarrollaran habilidades de manipulación de los mismos, así como para la realización de experimentos motivantes relacionados con la vida. En este sentido se trabajó de manera intencionada con las medidas de seguridad y las normas de trabajo en el laboratorio dada la inmadurez de la generalidad de los estudiantes y la tendencia asociada a ella de jugar con los útiles y reactivos y no cumplir con las normas para su uso adecuado.

El trabajo sistemático posibilitó el desarrollo del interés y la motivación de los estudiantes por la asignatura, ello posibilitó su participaron en la realización del inventario de los útiles de laboratorio y de las sustancias y reactivos químicos existentes en los IPU.

Se trabajó en el mejoramiento de la calidad de la preparación de las clases, al prestar atención sistemática al desarrollo de habilidades práctico- experimentales. En el sistema de evaluación se atendió de manera puntual a la medición de las habilidades, los conocimientos 
y los valores relacionados con la APE, tanto en evaluaciones sistemáticas como en parciales y final.

Se orientaron y ejecutaron trabajos teórico- prácticos de corte investigativo sobre las propiedades de las sustancias orgánicas, las reacciones exotérmicas y endotérmicas que ocurren en la vida y los factores que influyen en la velocidad de las reacciones químicas. En la realización de dichos trabajos se transitó de un uso deficiente de las fuentes de información y la copia textual e irreflexiva hacia el uso de fuentes de mayor actualización científica y la declaración de criterios que demuestran un aceptable nivel de comprensión de los contenidos.

Con la intención de abordar la FPE no solo desde el PEA se aprovechó el espacio del Proyecto socio-comunitario "Enigmas de la vida", que conducen los estudiantes de la licenciatura en educación en las carreras de Ciencias Naturales, para potenciar lo práctico experimental y establecer la relación con los contenidos de los programas de las asignaturas. El proyecto se ha desarrollado desde noviembre de 2015 , beneficiando a la comunidad escolar "La Edad de Oro". El mismo se ha orientado en dos direcciones principales: el vínculo de las actividades con el PEA de las Ciencias Naturales y el aprovechamiento de las potencialidades de la comunidad en función de estimular el aprendizaje y el interés de los estudiantes por dichas ciencias.

El trabajo del proyecto socio-comunitario partió de un diagnóstico integral de la comunidad, con la identificación de focos de contaminación, los centros de salud, las industrias, los centros de investigación, la situación de la agricultura urbana, otras instituciones educativas, la cría de animales y el cultivo de plantas. Así se determinaron las potencialidades y limitaciones para el estudio de las ciencias naturales y se proyectaron acciones en función de aprovechar las primeras y minimizar el efecto de las segundas.

Se habilitaron áreas de ciencias naturales en el Instituto tales como herbarios, terrarios, peceras, macetas, enriquecimiento de colecciones y se determinó cómo utilizar los mismos en el PEA de las referidas ciencias.

Se coordinó con la dirección de los IPU la participación de estudiantes en prácticas de laboratorio en la universidad, en particular, en dos que contribuyen al desarrollo de habilidades de uso frecuente en el laboratorio: determinar masa de muestras de sustancias químicas y medir volúmenes de líquidos. 


\section{Grupo II: en la formación de los licenciados en educación}

La introducción de los resultados del proyecto en la licenciatura en educación, especialidad Biología-Química estuvo dirigida al diseño y aplicación de las guías de orientación y evaluación de las prácticas de laboratorio de las asignaturas, en particular, las de las disciplinas Química Orgánica, Análisis Químico y Cristalografía, las mismas fueron suficientemente orientadoras para los estudiantes, sirviendo además de guía para su trabajo independiente durante la preparación previa y ejecución de las prácticas de laboratorio. Asimismo, se logró el desarrollo de habilidades intelectuales y prácticas- experimentales. Entre las primeras se destacan: observar, describir, interpretar y explicar los hechos y fenómenos en estudio; entre las segundas estuvieron: seleccionar y manipular útiles de laboratorio, masar muestras de sustancias químicas, preparar disoluciones, diseñar y montar aparatos de acuerdo con las propiedades de las sustancias reaccionantes y productos.

Se publicaron cuatro artículos científicos y se culminó un libro electrónico para la carrera de Biología-Química (También para la Licenciatura en Educación Química, correspondiente al nuevo plan de estudios E), de título: Concepción metodológica de las APE de las disciplinas de Química.

Con la introducción de los resultados en las disciplinas Didáctica de la Química y práctica laboral investigativa se logró que los estudiantes realizaran trabajos investigativos sobre la APE en la Química, los que sentaron las bases para su futuro trabajo de investigación. Se logró la presentación de sus trabajos en eventos.

En general la implementación de los resultados del proyecto en las asignaturas referidas, ha permitido:

1. El perfeccionamiento de sus preparaciones y mejoras en la calidad del PEA.

2. La elaboración de manuales didácticos, materiales de apoyo a la docencia, un libro electrónico y las guías de prácticas de laboratorio a realizar.

3. La publicación de artículos científicos relacionados con las APE y la FPE.

4. El desarrollo de habilidades asociadas a la FPE del estudiante.

5. El fortalecimiento del vínculo teoría-práctica a partir del incremento de APE.

6. El desarrollo de cuatro tesis doctorales y quince de maestría que se encuentran en proceso de realización. 
Los resultados obtenidos de la implementación en el PEA de las concepciones del proyecto se han socializado, con la participación protagónica de los estudiantes, en los eventos:

1. XXIX Fórum Nacional de Estudiantes de Ciencias Pedagógicas, noviembre de 2016

2. IV Encuentro Provincial de Círculos de Interés Pedagógicos, Sociedades Científicas y Grupos Científicos Estudiantiles. Octubre 2015 y 2016

3. Evento Provincial Pedagogía 2017. Ciego de Ávila. 28 de octubre de 2016.

4. Conferencia Científico Metodológica UNICA 2016. Universidad de Ciego de Ávila. 1 de septiembre de 2016.

5. XII Taller Nacional "Pedagogía". Centro de Estudio UNICA, Ciego de Ávila, 16 de junio de 2016.

6. I Encuentro Científico Nacional de Educación Ambiental y Desarrollo Sostenible 2016. Universidad Agraria de La Habana Fructuoso Rodríguez Pérez. Mayabeque. 26 y 27 de mayo de 2016.

7. IV Simposio Internacional de Vigilancia, Monitoreo y Control de vectores implicados en la transmisión de Enfermedades Zoonóticas. Febrero 2016.

8. Evento Provincial Didácticas de las Ciencias 2016. Dirección Provincial de Educación y Universidad de Ciego de Ávila Máximo Gómez Báez, 3 de noviembre de 2015.

9. Evento Provincial Universidad 2016, 10mo. Congreso Internacional de Educación Superior, en la Universidad de Ciego de Ávila Máximo Gómez Báez, 26 de junio de 2015.

Como insatisfacciones en la implementación de los resultados del proyecto se identifican:

1. Limitaciones en la atención al necesario vínculo de las acciones físicas y las intelectuales durante la ejecución de las APE; aún se pondera más la indicación de qué hacer y en menor medida se orienta sobre la reflexión y la argumentación de lo ocurrido.

2. Escasa utilización de la APE desde su concepción como problemas experimentales.

3. Poca intencionalidad de las APE para arribar a conclusiones a partir de la contrastación de los resultados obtenidos con los fundamentos teóricos de la ciencia.

4. Es limitado el tiempo que se utiliza para el trabajo en los laboratorios de las instituciones educativas. 


\section{Conclusiones}

La FPE supone un nivel de desarrollo de los estudiantes que se corresponda de manera directa y coherente con las intenciones de la enseñanza-aprendizaje de las ciencias naturales. Para que dicha formación se produzca debe darse el movimiento de lo externo a lo interno, del fenómeno a la esencia, de la dependencia a la independencia cognoscitiva, como camino dialéctico de la cognición que conduce a la comprensión de la naturaleza de la ciencia, y a asumir comportamientos y estilos de vida, en adecuada armonía con el medio social y natural.

La introducción de los resultados del proyecto se realizó a partir de intencionar el vínculo de las APE que se desarrollaron con la actividad investigativa y extensionista, aprovechando los recursos materiales y humanos de instituciones socioeconómicas existentes en la comunidad en función de la FPE de los estudiantes.

La evaluación de los resultados de la implementación del proyecto "La formación práctico-experimental en las ciencias naturales" se realizó mediante una metodología contentiva de cuatro etapas que permitió organizar, ejecutar y monitorear el proceso de introducción, posibilitando la recopilación de la información, su análisis, contrastación, socialización y la retroalimentación a los implicados en la experiencia sobre los logros y limitaciones que se obtuvieron.

El efecto logrado a partir de la introducción de los resultados del proyecto "Formación práctico experimental en las ciencias naturales" en el bachillerato y la licenciatura en educación se muestra en un incremento sostenido de la calidad de los trabajos teóricoprácticos y seminarios; los resultados de la evaluación del aprendizaje y las preparaciones de las asignaturas. Asimismo, se advierte la satisfacción de estudiantes, docentes, técnicos y directivos educacionales respecto al mejoramiento del desempeño de sus funciones. Se destaca el nivel de socialización de los resultados durante la ejecución del proyecto sociocomunitario. Aún no se ha logrado una adecuada correspondencia entre la realización de las operaciones manuales y el desarrollo de acciones intelectuales durante la ejecución de las APE. 


\section{Referencias}

Alexandre Dias, Bravo. (2011). La interdisciplinariedad de la metodología de la enseñanza de la Química con la Biología y la Geografía: una estrategia didáctica desde la actividad experimental, en la formación de profesores de la especialidad de Biología-Química de Viana, en Luanda, República de Angola (Tesis en opción al grado científico de Doctor en Ciencias Pedagógicas). U de Ciencias Pedagógicas "Enrique José Varona", La Habana, Cuba.

Arnaiz Barrios, Ibrahim. (2011). Dimensiones e indicadores para evaluar el impacto del proceso de introducción y generalización de resultados científicos en la práctica educativa (Informe de resultado científico de proyecto de investigación). Ciego de Ávila: Universidad de Ciencias Pedagógicas, Centro de Estudios e Investigaciones Educacionales.

Banasco Almentero, Josefa, Pérez Álvarez, Celina Esther, Pérez Capote, Manuel, Hernández Mujica, Jorge Lázaro, Caballero Camejo, Cayetano Alberto, Cuétara López, Ramón, Rico Molina, Pedro Pablo. (2013). Ciencias Naturales: una didáctica para su enseñanza y aprendizaje. La Habana: Editorial Pueblo y Educación.

Barraqué, Graciela. (1992). Metodología de la enseñanza de la Geografía. La Habana: Editorial de libros para la Educación.

Caballero Camejo, Cayetano y Vidal Tallet, Raúl. (febrero, 2014). La actividad práctica experimental de la química y el empleo de los software educativos como modo de actuación en la formación docente. Ponencia presentada en el VIII Congreso Didácticas de las Ciencias, La Habana, Cuba.

Castillo Estenoz, Micaela, Yera Quintana, Andrés Israel, Martínez Jiménez, Gerardo, Cruz Dávila, Maritza, Cárdenas Martínez, José Raúl y Rodríguez Frade Greidy. (2014). La formación práctico-experimental en las ciencias naturales (Informe de resultado de proyecto de investigación). Ciego de Ávila: Universidad de Ciencias Pedagógicas, Departamento de Ciencias Naturales.

Colado Pernas, José. (2003). Estructura didáctica para las actividades experimentales de las ciencias naturales en el nivel medio (Tesis en opción al grado científico de Doctor en Ciencias Pedagógicas), Universidad de Ciencias Pedagógicas Enrique José Varona, La Habana, Cuba.

Cuétara López, Ramón. (2004). Hacia una didáctica de la Geografía Local. La Habana: Editorial Pueblo y Educación.

García Cabrero, Benilde. (2010). Modelos teóricos e indicadores de evaluación educativa. Recuperado de http://www.scielo.org.mx/scielo.php?script=sci arttext\&pid=S1665109X2010000200005

Hedesa Pérez, Ysidro. (2015). Didáctica y currículo de la Química. La Habana: Editorial Pueblo y Educación. 
Hernández Sampieri, Roberto, Fernández Collado, Carlos y Baptista Lucio, Pilar. (2010). Metodología de la Investigación (5a․ ed) [Versión digital]. Recuperado de http://FreeLibros.com

Iglesias Triana, Leydis. (2012). Modelo didáctico para el perfeccionamiento del proceso de práctica de campo en la formación inicial de la carrera Biología-Geografía en la Universidad de Ciencias Pedagógicas "Rafael María de Mendive" (Tesis en opción al grado científico de Doctor en Ciencias Pedagógicas). Universidad de Ciencias Pedagógicas Rafael María de Mendive, Pinar del Río, Cuba.

Martínez Jiménez, Gerardo. (2009). El empleo de la tarea experimental en el proceso de enseñanza-aprendizaje de Ciencias Naturales en secundaria básica (Tesis en opción al grado científico de Doctor en Ciencias Pedagógicas). Universidad de Ciencias Pedagógicas Manuel Ascunce Domenech, Ciego de Ávila, Cuba.

Pérez Álvarez, Francisco y Hedesa Pérez, Ysidro. (2010). El experimento en el proceso de enseñanza-aprendizaje de la Química. Ponencia presentada en el VI Congreso Didácticas de las Ciencias, La Habana, Cuba.

Rionda Sánchez, Haydeé. (1999). La técnica semimicro en las actividades experimentales de la Química. La Habana: Editorial Pueblo y Educación.

Rodríguez Pérez, José. (2009). El experimento docente desarrollador. Un modelo didáctico de su dirección en la carrera de Profesor de Ciencias Naturales para la Educación Media Superior (Tesis en opción al grado científico de Doctor en Ciencias Pedagógicas). Universidad de Ciencias Pedagógicas Frank País García, Santiago de Cuba, Cuba.

Rojas Angel-Bello, Raidy. (2014). Metodología para la evaluación del impacto de los resultados científicos del proyecto de investigación (Informe de resultado de proyecto de investigación). Ciego de Ávila: Universidad de Ciencias Pedagógicas, Centro de Estudios e Investigaciones Educacionales.

Rojas Arce, Carlos y Achiong Caballero, Gustavo. (1990). El experimento químico y su papel en la realización de la función desarrolladora en la enseñanza. Ponencia presentada en el Congreso Internacional de Pedagogía 90, La Habana, Cuba.

Salcedo, Inés; Hernández, J.L.; Del Llano, M.R.;Mc Pherson, M. y Daudinot, I. (2002). Didáctica de la enseñanza de la Biología. La Habana: Editorial Pueblo y Educación.

Vidal Tallet, Raúl. (2012). La actividad práctico experimental de contenidos de Química con el apoyo de los software educativos en la formación inicial de profesores de BiologíaQuímica de la Educación Media. Una estrategia didáctica (Tesis en opción al grado científico de Doctor en Ciencias Pedagógicas). Universidad de Ciencias Pedagógicas Enrique José Varona, La Habana, Cuba.

Zilberstein Toruncha, José y Silvestre Oramas, Margarita. (2004). Didáctica integradora de las Ciencias Naturales. Experiencia cubana. La Habana: Editorial Academia. 


\section{Anexos}

\section{Anexo 1 \\ Criterios para la medición de las categorías evaluativas de los indicadores determinados para evaluar el impacto de la implementación del proyecto "Formación práctico experimental en las} ciencias naturales"

\begin{tabular}{|c|c|c|c|c|}
\hline Indicador & $\begin{array}{c}\text { Bastante } \\
\text { adecuado (4) }\end{array}$ & Adecuado (3) & $\begin{array}{c}\text { Poco adecuado } \\
\text { (2) }\end{array}$ & No adecuado (1) \\
\hline $\begin{array}{l}\text { Calidad de los } \\
\text { trabajos } \\
\text { teórico } \\
\text { prácticos y } \\
\text { seminarios }\end{array}$ & 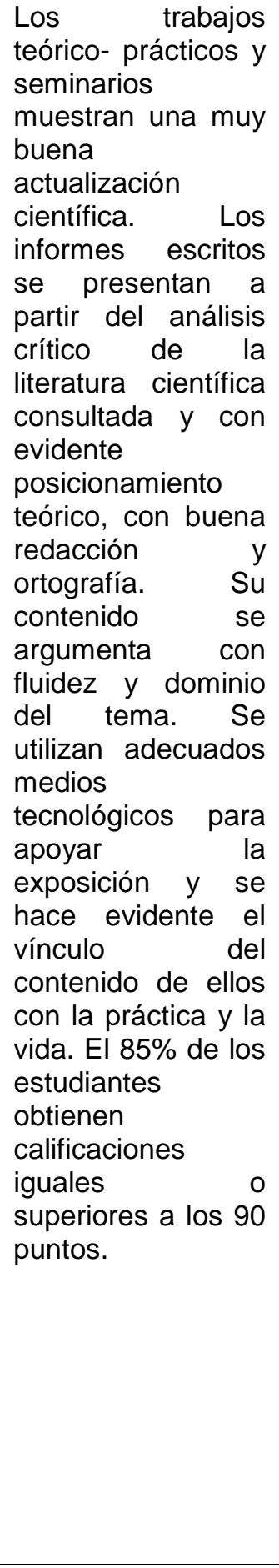 & 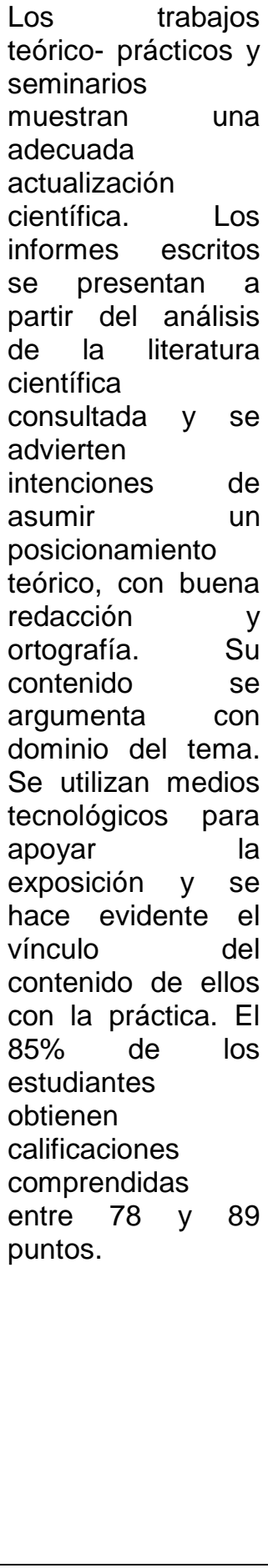 & 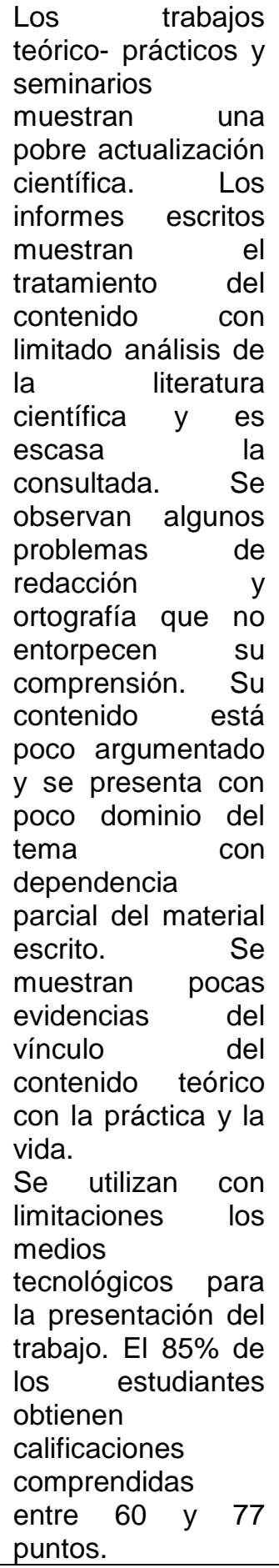 & 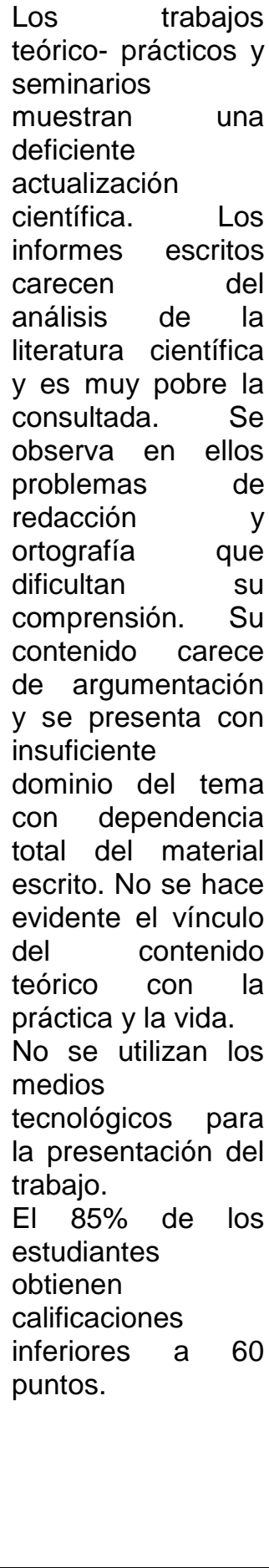 \\
\hline
\end{tabular}




\begin{tabular}{|c|c|c|c|c|}
\hline $\begin{array}{l}\text { Resultados de } \\
\text { la evaluación } \\
\text { del } \\
\text { aprendizaje }\end{array}$ & 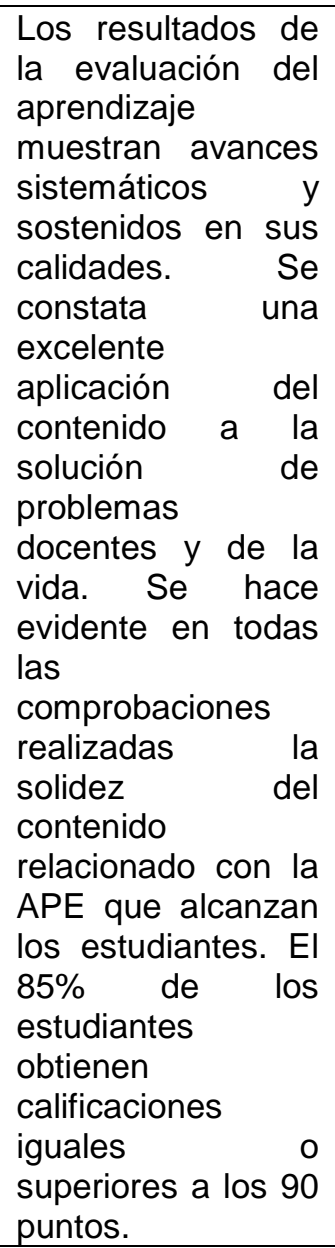 & $\begin{array}{l}\text { Los resultados de } \\
\text { la evaluación del } \\
\text { aprendizaje } \\
\text { muestran avances } \\
\text { en sus calidades. } \\
\text { Se constata una } \\
\text { buena aplicación } \\
\text { del contenido a la } \\
\text { solución de } \\
\text { problemas } \\
\text { docentes y de la } \\
\text { vida. Se hace } \\
\text { evidente en todas } \\
\text { las la } \\
\text { comprobaciones } \\
\text { parciales y finales } \\
\text { realizadas } \\
\text { solidez la } \\
\text { contenido } \\
\text { relacionado con la } \\
\text { APE que alcanzan } \\
\text { los estudiantes. El } \\
85 \% \text { de los } \\
\text { estudiantes } \\
\text { obtienen } \\
\text { calificaciones } \\
\text { comprendidas } \\
\text { entre } 78 \text { y } 89 \\
\text { puntos. }\end{array}$ & 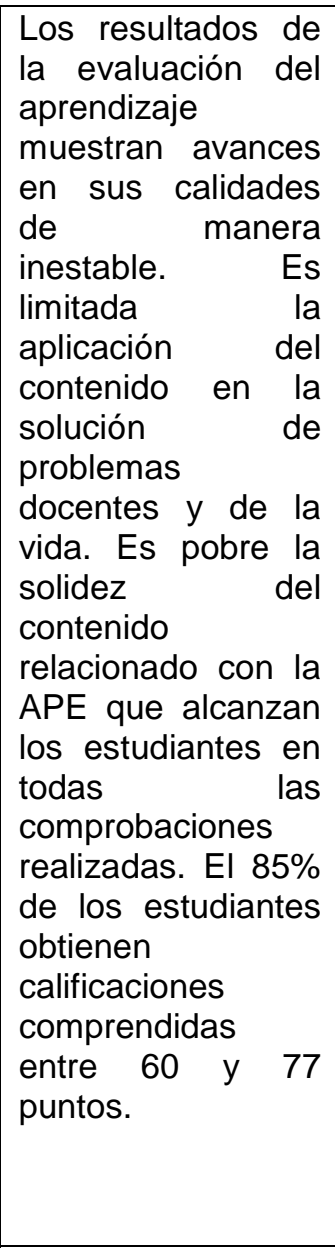 & $\begin{array}{l}\text { Los resultados de } \\
\text { la evaluación del } \\
\text { aprendizaje no } \\
\text { muestran avances } \\
\text { en sus calidades. } \\
\text { No se logra la } \\
\text { aplicación } \\
\text { contenido en lel } \\
\text { solución } \\
\text { problemas } \\
\text { docentes y de la } \\
\text { vida. Es deficiente } \\
\text { la solidez del } \\
\text { contenido } \\
\text { relacionado con la } \\
\text { APE que alcanzan } \\
\text { los estudiantes en } \\
\text { todas las } \\
\text { comprobaciones } \\
\text { realizadas. El } 85 \% \\
\text { de los estudiantes } \\
\text { obtienen } \\
\text { calificaciones } \\
\text { inferiores a } 60 \\
\text { puntos. }\end{array}$ \\
\hline \begin{tabular}{l}
\multicolumn{2}{l}{ Satisfacción } \\
de los \\
implicados \\
con \\
realización de \\
las APE
\end{tabular} & $\begin{array}{lr}\text { Existe una elevada } \\
\text { satisfacción } & \text { de } \\
\text { todos } & \text { los } \\
\text { involucrados en el } \\
\text { proyecto con la } \\
\text { realización de las } \\
\text { APE, la que se } \\
\text { manifiesta en el } \\
\text { aumento de sus } \\
\text { intereses por el } \\
\text { trabajo en el } \\
\text { laboratorio, su } \\
\text { disposición para } \\
\text { contribuir a su } \\
\text { mantenimiento y } \\
\text { cuidado. } \\
\text { sistemática } \\
\text { indagación sobre } \\
\text { nuevas la } \\
\text { experiencias que } \\
\text { puedan } \\
\text { desarrollarse para } \\
\text { lograr el vínculo } \\
\text { adecuado de la }\end{array}$ & $\begin{array}{lr}\begin{array}{l}\text { Existe } \\
\text { satisfacción }\end{array} & \text { buena } \\
\text { todos les } \\
\text { involucrados en el } \\
\text { proyecto con la } \\
\text { realización de las } \\
\text { APE, la que se } \\
\text { manifiesta en sus } \\
\text { intereses por el } \\
\text { trabajo en el } \\
\text { laboratorio y su } \\
\text { disposición para } \\
\text { contribuir a su } \\
\text { mantenimiento y } \\
\text { cuidado. } \\
\text { realizan se } \\
\text { indagaciones más } \\
\text { o menos } \\
\text { frecuentes sobre } \\
\text { nuevas } \\
\text { experiencias que } \\
\text { puedan } \\
\text { desarrollarse para } \\
\text { lograr el vínculo }\end{array}$ & $\begin{array}{l}\text { Es pobre la } \\
\text { satisfacción de los } \\
\text { involucrados en el } \\
\text { proyecto con la } \\
\text { realización de las } \\
\text { APE, la que se } \\
\text { manifiesta en un } \\
\text { inestable interés y } \\
\text { disposición por el } \\
\text { trabajo en el } \\
\text { laboratorio, } \\
\text { mantenimiento su } \\
\text { cuidado. } \\
\text { desarrollan } \\
\text { indagaciones sobre } \\
\text { nuevas } \\
\text { experiencias que } \\
\text { puedan } \\
\text { desarrollarse para } \\
\text { lograr el vínculo } \\
\text { adecuado de la } \\
\text { teoría con la } \\
\text { práctica, solo en } \\
\text { los casos en que }\end{array}$ & $\begin{array}{l}\text { Es insuficiente la } \\
\text { satisfacción de los } \\
\text { involucrados en el } \\
\text { proyecto con la } \\
\text { realización de las } \\
\text { APE, la que se } \\
\text { manifiesta en un } \\
\text { pobre interés y } \\
\text { baja disposición } \\
\text { por el trabajo en el } \\
\text { laboratorio y su } \\
\text { mantenimiento y } \\
\text { cuidado. No } \\
\text { constituye interés } \\
\text { la indagación sobre } \\
\text { nuevas que } \\
\text { experiencias que } \\
\text { puedan lan } \\
\text { desarrollarse para } \\
\text { lograr el vínculo } \\
\text { adecuado de la } \\
\text { teoría con la } \\
\text { práctica. }\end{array}$ \\
\hline
\end{tabular}




\begin{tabular}{|c|c|c|c|c|}
\hline & $\begin{array}{lll}\text { teoría } & \text { con } & \text { la } \\
\text { práctica. } & & \end{array}$ & 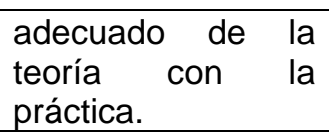 & $\begin{array}{l}\text { ellas son indicadas } \\
\text { por el docente. }\end{array}$ & \\
\hline $\begin{array}{ll}\text { Utilización de } \\
\text { materiales } & \\
\text { docentes } & \\
\text { elaborados } & \\
\text { por } & \text { el } \\
\text { proyecto } & \end{array}$ & $\begin{array}{lr}\text { Se utilizan } & \text { con } \\
\text { sistematicidad } & \text { los } \\
\text { manuales } & \\
\text { didácticos, guías } \\
\text { de prácticas de } \\
\text { laboratorio } & \text { y } \\
\text { materiales } & \text { de } \\
\text { apoyo a la } \\
\text { docencia } \\
\text { elaborados por el } \\
\text { proyecto y se } \\
\text { contribuye a su } \\
\text { perfeccionamiento } \\
\text { constante a partir } \\
\text { de los criterios } \\
\text { fundamentados } \\
\text { que se emiten por } \\
\text { los que los utilizan. }\end{array}$ & $\begin{array}{lr}\text { Se utilizan } & \text { con } \\
\text { sistematicidad } & \text { los } \\
\text { manuales } & \\
\text { didácticos, guías } \\
\text { de prácticas } \\
\text { laboratorio de } \\
\text { materiales } & \text { y } \\
\text { apoyo a la } \\
\text { docencia a } \\
\text { elaborados por el } \\
\text { proyecto y s se } \\
\text { contribuye a su } \\
\text { perfeccionamiento } \\
\text { sistemático a partir } \\
\text { de los criterios } \\
\text { emitidos por los } \\
\text { que los utilizan. }\end{array}$ & $\begin{array}{lr}\text { Se utilizan poco los } \\
\text { manuales } \\
\text { didácticos, guías } \\
\text { de prácticas } \\
\text { laboratorio } & \text { y } \\
\text { materiales } & \text { de } \\
\text { apoyo a la } \\
\text { docencia } & \\
\text { elaborados por el } \\
\text { proyecto y se } \\
\text { contribuye poco a } \\
\text { su } \\
\text { perfeccionamiento } \\
\text { a partir de escasos } \\
\text { criterios emitidos } \\
\text { por los que los } \\
\text { utilizan. }\end{array}$ & $\begin{array}{lr}\text { Se utilizan } & \text { muy } \\
\text { escasamente } & \text { los } \\
\text { manuales } & \\
\text { didácticos, guías } \\
\text { de prácticas } \\
\text { laboratorio } \\
\text { materiales } \\
\text { apoyo a de la } \\
\text { docencia } \\
\text { elaborados por el } \\
\text { proyecto y no se } \\
\text { contribuye a su } \\
\text { perfeccionamiento } \\
\text { por carecer de los } \\
\text { criterios que de su } \\
\text { uso se pueden } \\
\text { emitir. }\end{array}$ \\
\hline $\begin{array}{l}\text { Calidad de las } \\
\text { preparaciones } \\
\text { de las } \\
\text { asignaturas }\end{array}$ & 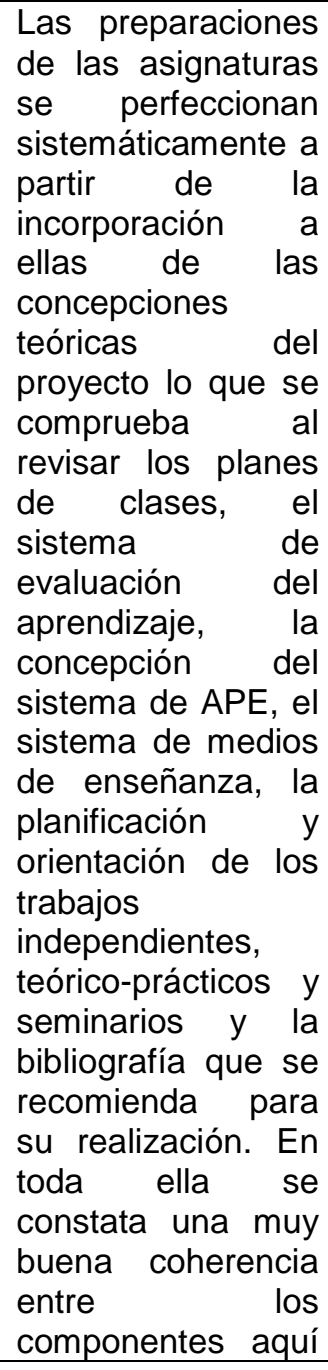 & 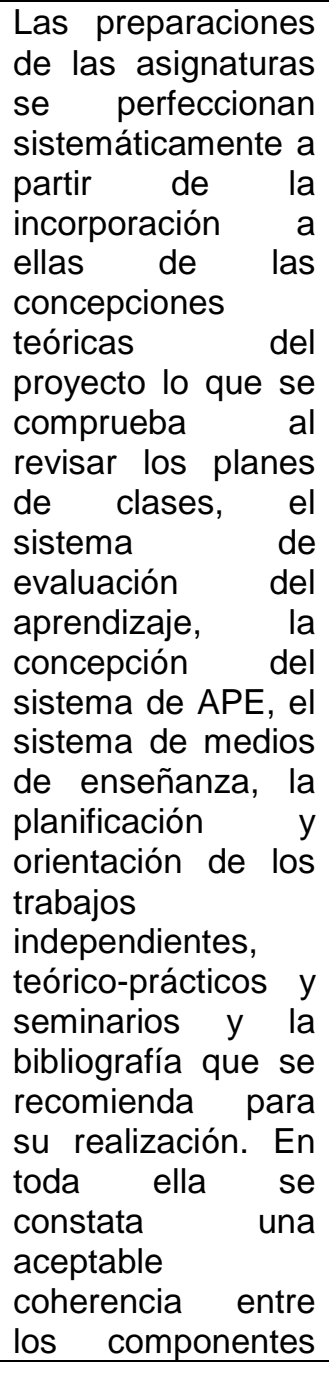 & 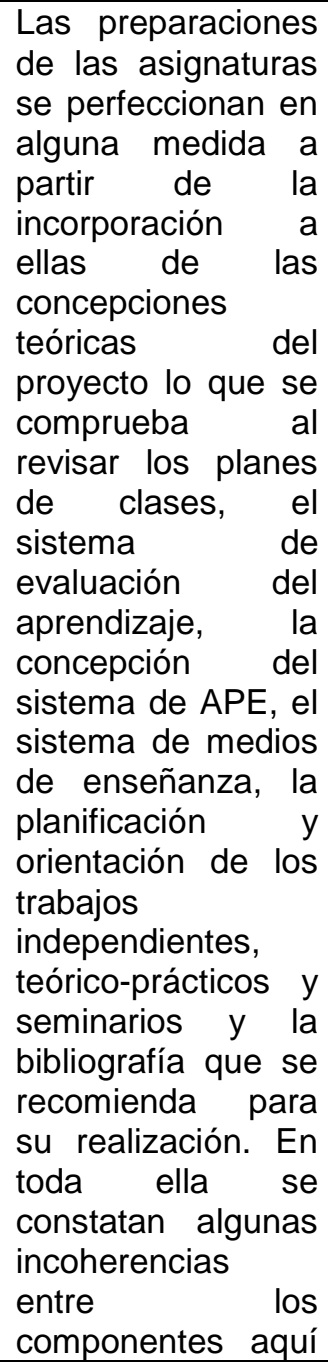 & $\begin{array}{lr}\text { Las preparaciones } \\
\text { de las asignaturas } \\
\text { no se perfeccionan } \\
\text { a partir de la } \\
\text { incorporación } \\
\text { ellas de las } \\
\text { concepciones } \\
\text { teóricas del } \\
\text { proyecto lo que se } \\
\text { comprueba al } \\
\text { revisar los planes } \\
\text { de clases, el } \\
\text { sistema de } \\
\text { evaluación del } \\
\text { aprendizaje, la } \\
\text { concepción del } \\
\text { sistema de APE, el } \\
\text { sistema de medios } \\
\text { de enseñanza, la } \\
\text { planificación y } \\
\text { orientación de los } \\
\text { trabajos } \\
\text { independientes, } \\
\text { teórico-prácticos y } \\
\text { seminarios y la } \\
\text { bibliografía que se } \\
\text { recomienda para } \\
\text { su realización. En } \\
\text { toda ella se } \\
\text { constata falta de } \\
\text { coherencia entre } \\
\text { los componentes } \\
\text { aquí declarados. } \\
\text { En los documentos }\end{array}$ \\
\hline
\end{tabular}




\begin{tabular}{|c|c|c|c|c|}
\hline & \begin{tabular}{lr} 
declarados. \\
En todos los \\
documentos que \\
forman parte de \\
dicha preparación \\
de constatan \\
anotaciones y \\
comentarios \\
fundamentados de \\
los docentes con \\
vistas \\
mejoramiento de \\
\multicolumn{2}{l}{ sus calidades. } \\
\end{tabular} & $\begin{array}{lr}\text { aquí declarados. } \\
\text { En todos los } \\
\text { documentos que } \\
\text { forman parte de } \\
\text { dicha preparación } \\
\text { de constatan } \\
\text { anotaciones y } \\
\text { comentarios de los } \\
\text { docentes con } \\
\text { vistas al } \\
\text { mejoramiento de } \\
\text { sus calidades. }\end{array}$ & $\begin{array}{lr}\text { declarados. } \\
\text { En los documentos } \\
\text { que forman parte } \\
\text { de } & \text { dicha } \\
\text { preparación } & \text { se } \\
\text { constatan escasas } \\
\text { anotaciones } & \text { y } \\
\text { comentarios de los } \\
\text { docentes r con } \\
\text { vistas } & \text { al } \\
\text { mejoramiento } & \text { de } \\
\text { sus calidades. }\end{array}$ & $\begin{array}{lr}\text { que forman } & \text { parte } \\
\text { de } & \text { dicha } \\
\text { preparación } & \text { no se } \\
\text { constatan } & \\
\text { anotaciones } & \text { ni } \\
\text { comentarios } & \text { de los } \\
\text { docentes } & \text { con } \\
\text { vistas } & \text { al } \\
\text { mejoramiento } & \text { de } \\
\text { sus calidades. } & \end{array}$ \\
\hline $\begin{array}{lr}\text { Nivel } & \text { de } \\
\text { aplicación } & \text { del } \\
\text { resultado } & \end{array}$ & 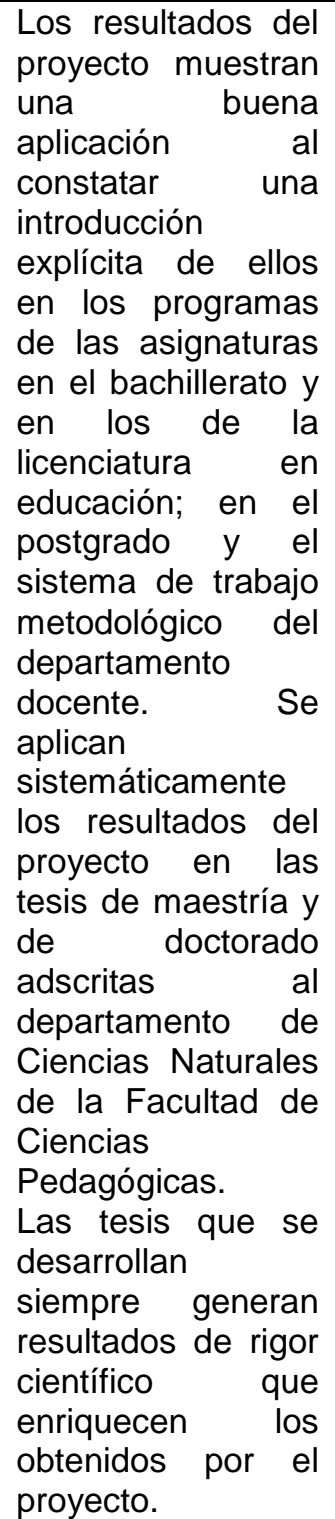 & 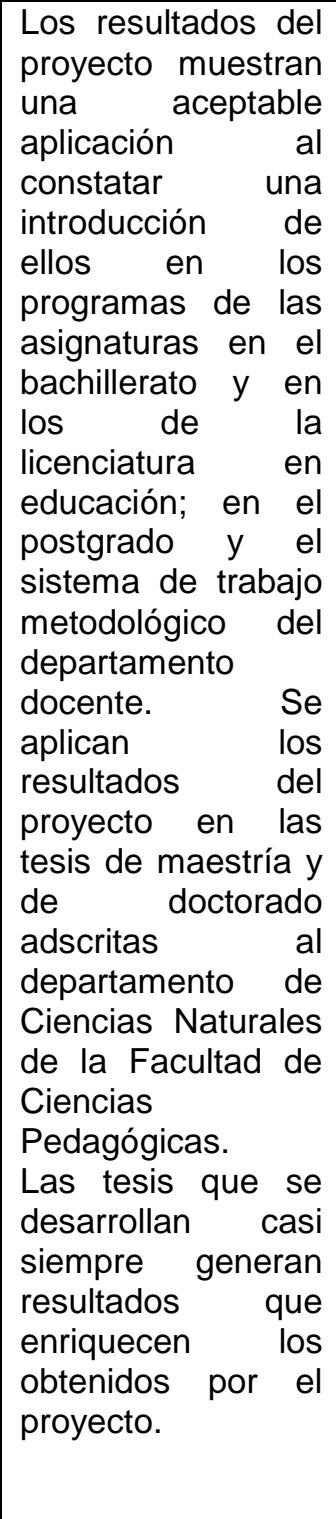 & 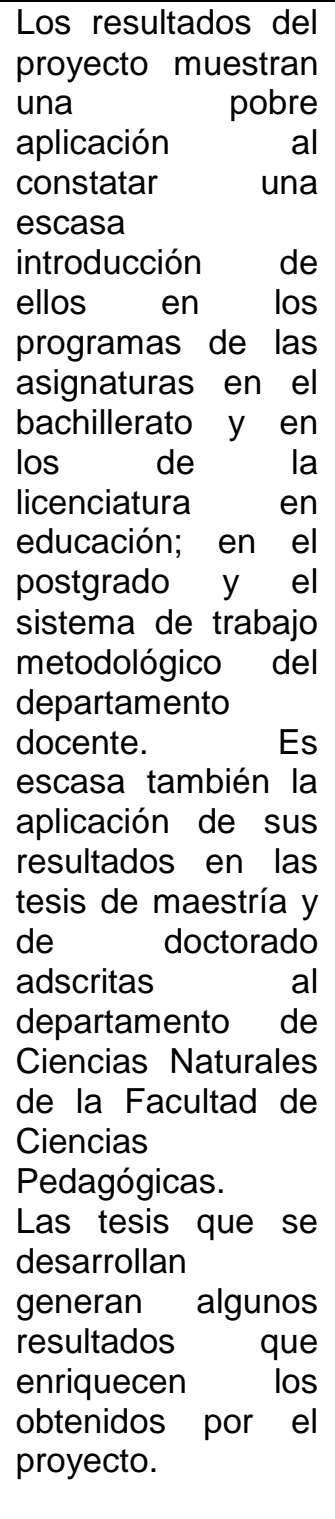 & 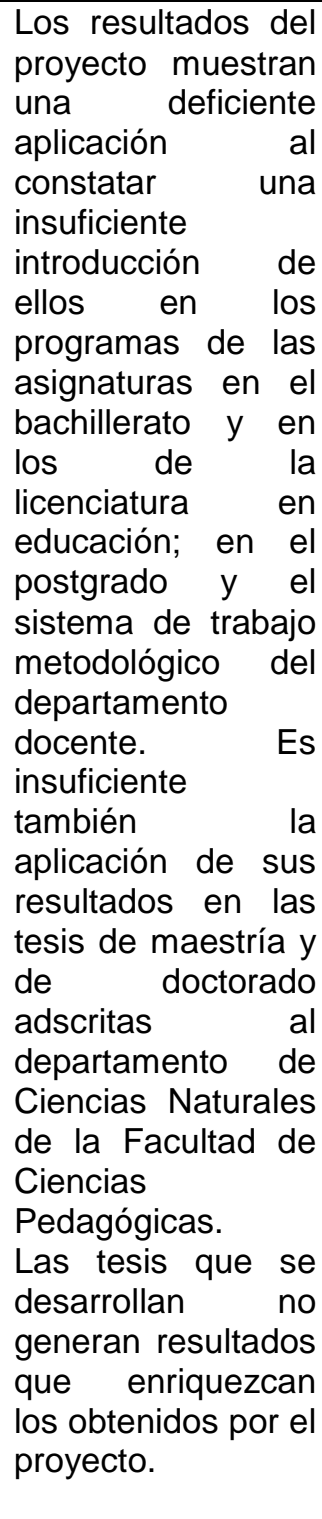 \\
\hline $\begin{array}{l}\text { Nivel de } \\
\text { socialización } \\
\text { de los } \\
\text { resultados }\end{array}$ & \begin{tabular}{lr} 
Se & \multicolumn{2}{r}{ socializan } \\
ampliamente & los \\
resultados & del \\
proyecto & en
\end{tabular} & $\begin{array}{lr}\text { Se socializan } & \text { los } \\
\text { resultados } & \text { del } \\
\text { proyecto } & \text { en } \\
\text { eventos locales, }\end{array}$ & $\begin{array}{lr}\text { Se } & \text { socializan } \\
\text { escasamente } & \text { los } \\
\text { resultados } & \text { del } \\
\text { proyecto } & \text { en }\end{array}$ & $\begin{array}{lr}\text { Se } & \text { socializan } \\
\text { escasamente } & \text { los } \\
\text { resultados } & \text { del } \\
\text { proyecto } & \text { en }\end{array}$ \\
\hline
\end{tabular}




\begin{tabular}{|c|c|c|c|c|}
\hline & 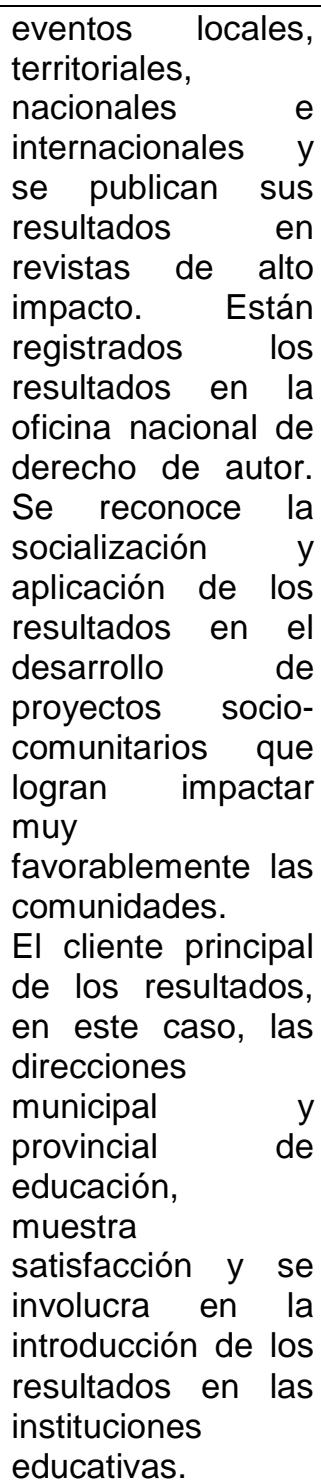 & 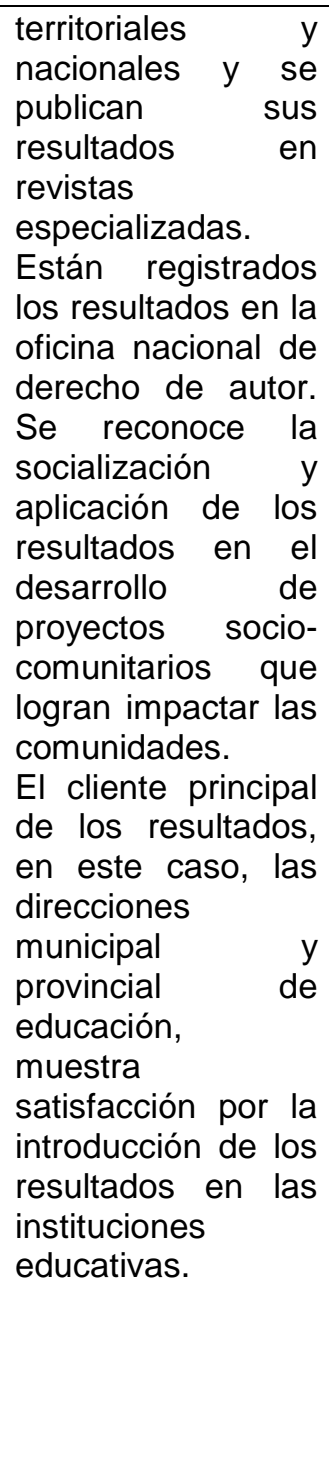 & 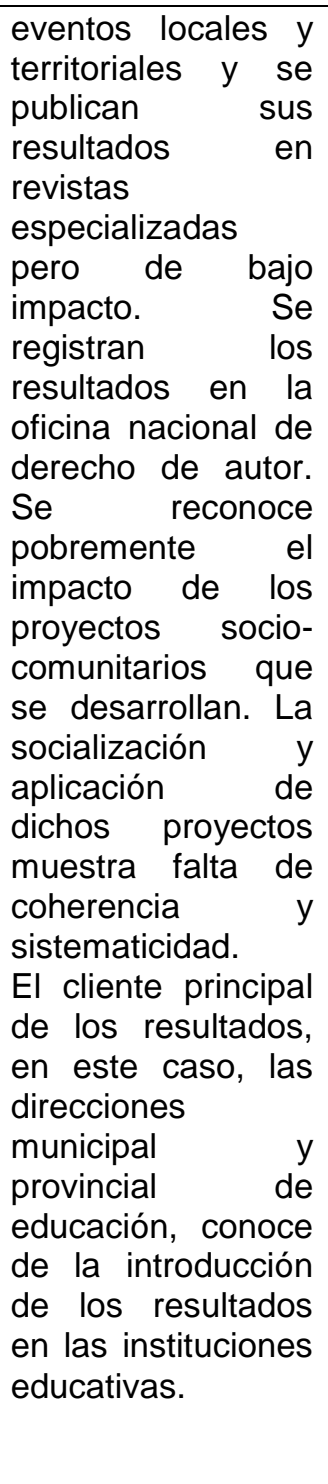 & $\begin{array}{l}\text { eventos locales y } \\
\text { no se publican sus } \\
\text { resultados. No se } \\
\text { registran los } \\
\text { resultados en la } \\
\text { oficina nacional de } \\
\text { derecho de autor. } \\
\text { No se reconoce el } \\
\text { impacto de los } \\
\text { proyectos socio- } \\
\text { comunitarios que } \\
\text { se desarrollan. La } \\
\text { socialización y } \\
\text { aplicación de } \\
\text { dichos proyectos } \\
\text { es insuficiente. } \\
\text { El cliente principal } \\
\text { de los resultados, } \\
\text { en este caso, las } \\
\text { direcciones } \\
\text { municipal } \\
\text { provincial y } \\
\text { educación, de se } \\
\text { muestra ajeno a la } \\
\text { introducción de los } \\
\text { resultados en las } \\
\text { instituciones } \\
\text { educativas. }\end{array}$ \\
\hline $\begin{array}{l}\text { Apertura de } \\
\text { nuevas líneas } \\
\text { y/o tareas de } \\
\text { investigación }\end{array}$ & $\begin{array}{lr}\text { Se logra la } \\
\text { apertura de nuevas } \\
\text { líneas y tareas de } \\
\text { investigación a } \\
\text { partir de las } \\
\text { contribuciones de } \\
\text { los investigadores } \\
\text { y clientes del } \\
\text { proyecto. } \\
\text { La incorporación } \\
\text { de las nuevas } \\
\text { líneas y tareas } \\
\text { están a tono con } \\
\text { los debates y } \\
\text { análisis generados } \\
\text { en las sesiones de } \\
\text { trabajo dy del } \\
\text { proyecto y los }\end{array}$ & $\begin{array}{lr}\text { Se logra la } \\
\text { apertura de nuevas } \\
\text { tareas } & \text { de } \\
\text { investigación } & \text { a } \\
\text { partir de las } \\
\text { contribuciones de } \\
\text { los investigadores } \\
\text { del proyecto. } \\
\text { La incorporación } \\
\text { de las nuevas } \\
\text { tareas r r } \\
\text { corresponde con } \\
\text { los debates y } \\
\text { análisis generados } \\
\text { en las sesiones de } \\
\text { trabajo r del } \\
\text { proyecto y los } \\
\text { talleres y defensas }\end{array}$ & \begin{tabular}{lr} 
Se & \multicolumn{2}{c}{ constatan } \\
intentos para la \\
apertura de nuevas \\
tareas \\
investigación de a \\
partir de los \\
debates y análisis \\
desarrollados en el \\
proyecto. \\
Existe cierta \\
claridad de los \\
resultados que se \\
esperan pero no de \\
la manera en que \\
pudieran impactar \\
en \\
perfeccionamiento \\
de la FPE de los
\end{tabular} & $\begin{array}{l}\text { No se logra la } \\
\text { apertura de nuevas } \\
\text { líneas y tareas de } \\
\text { investigación. } \\
\text { Los debates y } \\
\text { análisis generados } \\
\text { por el proyecto } \\
\text { solo se encaminan } \\
\text { a evaluar el } \\
\text { cumplimiento o no } \\
\text { de las tareas } \\
\text { planificadas. } \\
\text { No existe claridad } \\
\text { de los resultados } \\
\text { que se esperan ni } \\
\text { de la manera en } \\
\text { que pueden } \\
\text { impactar en el }\end{array}$ \\
\hline
\end{tabular}




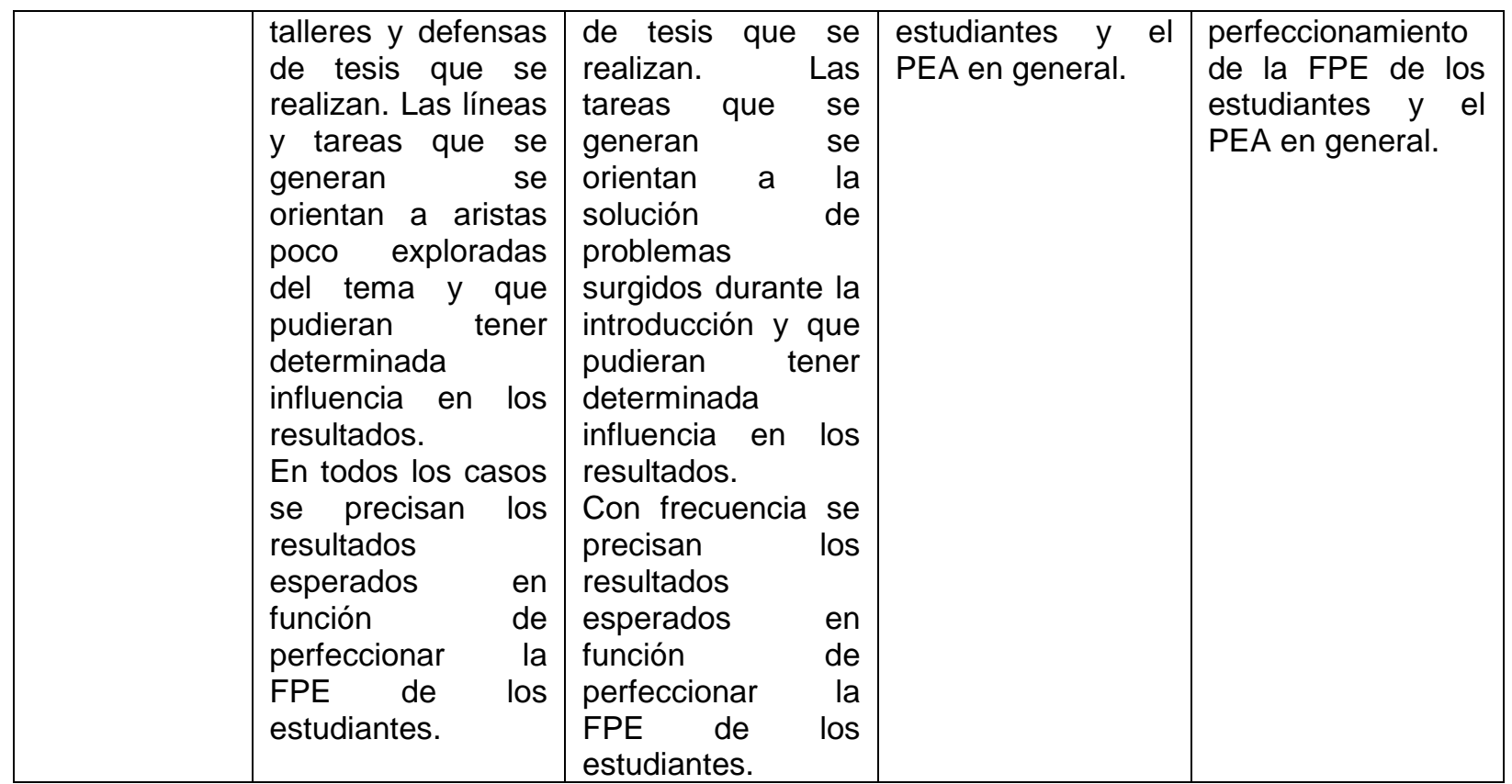

